gen molecules on human granulocytes during early phases of differentiation. Proc Natl Acad Sci U S A. 1977;74(9):4012-4016.

3. Guba SC, Stella G, Turka LA, June CH, Thompson $\mathrm{CB}$, Emerson SG. Regulation of interleukin 3 gene induction in normal human $\mathrm{T}$ cells. J Clin Invest. 1989;84(6):1701-1706

4. Hexner EO, et al. Umbilical cord blood xenografts in immunodeficient mice reveal that $\mathrm{T}$ cells enhance hematopoietic engraftment beyond overcoming immune barriers by stimulating stem cell differentiation. Biol Blood Marrow Transplant. 2007;13(10):1135-1144

5. Essers MA, et al. IFNalpha activates dormant haematopoietic stem cells in vivo. Nature. 2009; 458(7240):904-908

6. Baldridge MT, King KY, Boles NC, Weksberg DC, Goodell MA. Quiescent haematopoietic stem cells are activated by IFN-gamma in response to chronic infection. Nature. 2010;465(7299):793-797.
7. Luna G, Paez J, Cardier JE. Expression of the hematopoietic stem cell antigen Sca-1 (LY-6A/E) in liver sinusoidal endothelial cells: possible function of Sca-1 in endothelial cells. Stem Cells Dev. 2004;13(5):528-535.

8. Chen C, Liu Y, Liu R, Ikenoue T, Guan KL, Zheng P. TSC-mTOR maintains quiescence and function of hematopoietic stem cells by repressing mitochondrial biogenesis and reactive oxygen species. J Exp Med. 2008;205(10):2397-2408.

\title{
The cellular response to hypoxia: tuning the system with microRNAs
}

\author{
Joseph Loscalzo \\ Department of Medicine, Brigham and Women’s Hospital, Harvard Medical School, Boston, Massachusetts, USA.
}

\begin{abstract}
Adaptation to hypoxia is an essential cellular response controlled by the oxygen-sensitive master transcription factor hypoxia-inducible factor 1 (HIF-1). HIF-1 expression is also controlled by specific microRNAs and, in turn, controls the expression of other microRNAs, which fine-tune adaptation to low oxygen tension. In this issue of the JCI, Ghosh and colleagues identify a unique microRNA in hypoxic endothelial cells, miR424, that promotes HIF-1 stabilization and angiogenesis. The actions of this microRNA are considered in the context of the complex interactions that act to ensure optimal endothelial adaptation to this critical environmental condition.
\end{abstract}

Adaptation to hypoxia is an essential homeostatic mechanism in mammalian cells. Hypoxia can occur not only as a global consequence of low atmospheric oxygen tension, but also locally at sites of inflammation, tissue ischemia and injury, and solid tumor growth. Fundamental mechanisms have, therefore, evolved by which cells adapt to hypoxic conditions and their potentially adverse consequences, both acutely and chronically, in health and disease.

Among the key responses to acute hypox$i a$ is the upregulation of hypoxia-inducible factors (HIFs), master transcription factors that induce the highly conserved expression of many genes (likely more than 100) and are responsible for the majority of the hypoxic program in metazoan cells (1). The prototypical HIF is a heterodimer of $\alpha$ and $\beta$ subunits. Under normoxic conditions, the $\alpha$ subunit is rapidly hydroxylated at specific prolyl residues by prolyl hydroxylase domain (PHD) proteins. Upon hydroxylation, the $\alpha$ subunit is recognized by the von HippelLindau protein and consequently undergoes rapid proteasomal degradation. Under

Conflict of interest: The author has declared that no conflict of interest exists.

Citation for this article: J Clin Invest. 2010; 120(11):3815-3817. doi:10.1172/JCI45105. hypoxic conditions, however, the $\alpha$ subunit does not undergo prolyl hydroxylation and, as a result, forms a stable complex with the $\beta$ subunit, which binds as a heterodimer to hypoxia response elements (HREs) in the promoter regions of hypoxia-sensitive genes to induce gene transcription. HIF-dependent transcriptional changes lead to a broad range of cellular adaptations, including metabolic, proliferative, apoptotic, and angiogenic. In higher metazoans, three different HIF- $\alpha$ proteins have been identified: HIF- $1 \alpha$ and HIF- $2 \alpha$ have some transcriptional targets in common and some that are unique to each subunit, while splice variants of HIF-3 $\alpha$ have dominant negative effects on HIFdependent gene transcription $(2,3)$.

\section{MicroRNAs and transcriptional tuning}

MicroRNA molecules (miRNAs) are essential, noncanonical RNA species of 18-23 nucleotides in length that regulate gene expression. There are believed to be more than 1,000 miRNA genes in the human genome, which are estimated to regulate more than onethird of all mRNA transcripts (4). As part of the RNA-induced silencing complex (RISC), miRNAs negatively regulate gene transcription by annealing to the $3^{\prime}$ untranslated region of specific mRNA targets to repress translation, enhance mRNA degradation, or both. In contrast to the promoter-based catalytic amplification of mRNA synthesis, miRNAs are viewed as weak modulators of the transcriptional response, fine-tuning gene expression largely by negative regulation in a process that requires stoichiometric binding to mRNA targets (5).

\section{Hypoxamirs: microRNAs induced by hypoxia}

Hypoxia has recently been shown to induce the expression of a number of miRNAs, which have been termed "hypoxamirs" (6). Owing to the central importance of HIFs to the hypoxic response, its potent regulation of many genes whose expression is regulated by oxygen tension, and the significant number of hypoxamirs, it is logical to explore the relationships among HIFs and hypoxamirs in order to understand better the complex regulation of the hypoxic program in metazoans.

From the perspective of the miRNA response to hypoxia, miRNAs can be viewed as comprising three groups (Table 1): first, those induced by HIFs under hypoxic conditions (HIF-dependent hypoxamirs); second, those induced by hypoxia that, in turn, affect expression of HIF; third, those whose expression is not dependent on hypoxia but that affect HIF expression. The number of hypoxamirs that have been identified in each of these groups is expanding rapidly, illustrating the extraordinary regulatory complexity of the hypoxic program and the importance of considering that program in a holistic, system-based context. In this issue of the JCI, Ghosh and colleagues (7) identify a new hypoxamir, miR424, that affects HIF expression. This report is unique for three reasons: it is the first demonstration of the 
Table 1

Hypoxia, microRNAs, and HIF

\section{Hypoxamirs induced by HIF}

$\operatorname{miR} 210$

miR373
Hypoxamirs that affect HIF

miR20b

miR199a

miR424

\section{miRNAs that affect HIF independent of hypoxia miR107 miR17-92 cluster miR31 \\ miR519c}

hypoxic induction of this microRNA, as a prior study showed that hypoxia suppressed its expression in trophoblasts (8); it is the first demonstration of a hypoxamir unique to the endothelial cell; and it enhances HIF stability in a novel way (see below).

Among the HIF-dependent hypoxamirs are $\operatorname{miR} 210$ and miR373 $(7,8)$. While miR373 has not been studied in great detail (9), miR210 has been extensively studied in its role as a hypoxamir (8-13). The most robustly induced hypoxamir, miR 210 , is induced by HIF-1 $\alpha$ (10) and suppresses expression of the cell-cycle regulator E2F transcription factor 3 (E2F3) (11), the receptor tyrosine kinase ligand ephrin A3 (12), and the DNA repair protein RAD52 (13). miR210 was recently also shown to have the important role of suppressing mitochondrial metabolism in hypoxic states by decreasing expression of the iron-sulfur cluster assembly proteins ISCU $1 / 2$, thereby limiting cytochrome assembly and ROS generation from inefficient mitochondrial electron transport under low oxygen tensions $(14,15)$. This observation adds to the other known effects of HIF-1 on mitochondrial metabolism. These include suppression of ROS generation under hypoxic conditions by switching expression of the regulatory subunit of cytochrome $c$ oxidase from COX4 to COX2, which increases the efficiency of complex IV; induction of pyruvate dehydrogenase 1, which shunts pyruvate from the mitochondrion; and induction of BCL2/adenovirus E1B 19-kDa interacting protein 3 (BNIP3), which triggers mitophagy (16).

Three hypoxamirs have been shown to affect HIF expression: miR20b, miR199a, and, most recently, miR424. miR20b targets HIF- $1 \alpha$ to suppress its expression (in MCF-7 breast cancer cells) (17), and downregulation of miR199a derepresses HIF-1 $\alpha$ (in cardiomyocytes) (18). Ghosh and colleagues show that miR424 regulates HIF- $\alpha$ isoforms in endothelial cells by targeting cullin 2 (CUL2), the scaffolding protein on which the ubiquitin ligase system assembles, there- by stabilizing HIF- $\alpha$ isoforms by impairing their prolyl hydroxylation (7). miR424 itself is induced by hypoxia via PU.1 transactivation, levels of which are increased in hypoxic endothelial cells by runt-related transcription factor 1 (RUNX-1) and CCAAT/enhancer binding protein $\alpha(\mathrm{C} / \mathrm{EBP} \alpha)(7)$.

In addition to these hypoxamir-mediated responses, at least four miRNAs have been shown to influence HIF expression independent of hypoxia. Induced by p53, miR107 decreases expression of HIF- $\beta$ (19); induced by c-MYC, the miR17-92 cluster suppresses expression of HIF-1 $\alpha$ (20); and suppressed by hepatocyte growth factor, miR519c suppresses expression of HIF-1 $\alpha$ (21). In contrast, miR31, by decreasing expression of the HIF regulatory factor factor-inhibiting HIF $(\mathrm{FIH})$, increases expression of HIF-1 $\alpha$ (22).

\section{Hypoxamir expression and cellular context}

It is important to consider the cellular context within which hypoxamirs are expressed. The upregulation of miR 210 is robust and has been observed in all hypoxic cell types in which it has been studied (14). In contrast, the upregulation of miR424 reported by Ghosh and colleagues (7) is unique to the endothelial cell. The reason for this distinction is not entirely clear, but may reflect the central importance of the endothelium at the interface of flowing blood and metabolizing tissue. Poised at this interface, the endothelium is uniquely situated to sense hypoxia upon blood flow reduction and to initiate the reparative angiogenic response required for its restoration. The endothelium has great metabolic plasticity, acquiring a significant fraction of its ATP needs from glycolysis; HIF-1 $\alpha$ regulates this shift from oxidative phosphorylation to anaerobic glycolysis under hypoxic conditions and does so in conjunction with reducing mitochondrial number (23) and function (14). The induction of miR424 in endothelial cells prolongs the action of HIF- $1 \alpha$, which would be expected to promote further glycolytic metabolism and limit ROS-mediated cytotoxicity. In this way, miR424 is an important regulator of the durability of the hypoxic HIF program. In addition and importantly, miR424 is induced by another potent transcription factor, PU.1, levels of which increase in hypoxic endothelium (7). This transcription factor working in concert with miR424 has been shown to regulate monocyte differentiation (24); miR424 has also been shown to regulate monocyte differentiation by combinatorial interactions with $\mathrm{miR} 155$, miR222, and miR503 (25). Whether miR424 also regulates endothelial cell differentiation from precursor cells is an unanswered question that would add another dimension to the action of this pleiotropic miRNA and would clearly complement its indirect, HIF-1 $\alpha$-dependent effects on angiogenesis so essential for restoring tissue perfusion and oxygen delivery.

\section{Conclusion}

Clearly, the response to hypoxia is complex and driven by a network-based system with various levels of interaction and feedback. Hypoxamirs interacting with HIF-driven responses add a combinatorial richness to

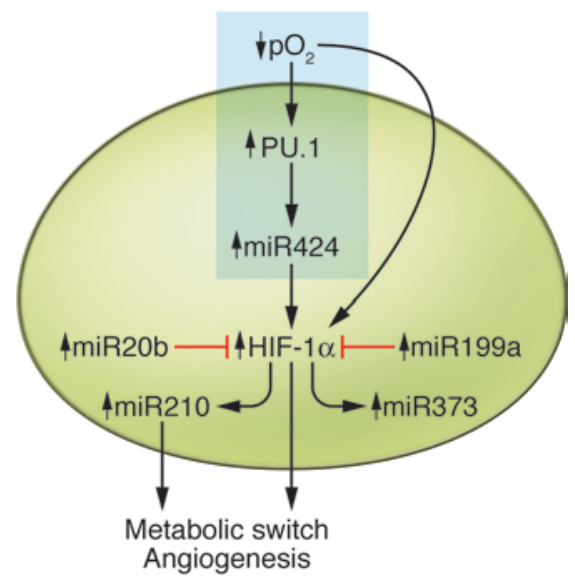

\section{Figure 1}

The endothelial cell response to hypoxia. The endothelium is poised between flowing blood and metabolizing tissue. Under the hypoxic (ischemic) conditions accompanying reductions in blood flow, the endothelial response is governed by HIF, as well as hypoxamirs, which are themselves either induced by HIF or affect HIF expression and action. The net effect is a metabolic switch to glycolysis and endothelial proliferation and differentiation, actions that facilitate durable adaptation to hypoxia sufficient to promote angiogenesis and restore blood flow. The text highlighted in the blue rectangle indicates how the work of Ghosh and colleagues integrates with current understanding of hypoxamirs. 
this system that defines the tissue context of the hypoxic program and provides a method for tuning the responses of transcription factors under the wide range of conditions that contribute to the genesis of hypoxic stress. The paper by Ghosh and colleagues (7) adds yet another dimension to this response by demonstrating the HIFindependent hypoxic induction of a hypoxamir unique to the endothelium, which prolongs HIF expression and thereby promotes the hypoxic induction of the angiogenic response required to restore tissue perfusion and attenuate tissue hypoxia (Figure 1). It is an elegant system of extraordinary complexity that is essential for homeostatic control under variable oxygen tensions.

\section{Acknowledgments}

This work was supported in part by NIH grants HL61795, HL81587, HL70819, and HL48743. The author wishes to thank Stephanie Tribuna for expert technical assistance.

Address correspondence to: Joseph Loscalzo, Department of Medicine, Brigham and Women's Hospital, 75 Francis Street, Boston, Massachusetts 02115-2394, USA. Phone: 617.732.6340; Fax: 617.732.6439; E-mail: jloscalzo@partners.org.

1. Kaelin WG Jr, Ratcliffe PJ. Oxygen sensing by metazoans: the central role of the HIF hydroxylase pathway. Mol Cell. 2008;30(4):393-402.

2. Wang V, Davis DA, Haque M, Huang LE, Yarchoan R.
Differential gene up-regulation by hypoxiainducible factor-1alpha and hypoxia-inducible factor-2alpha in HEK293T cells. Cancer Res. 2005;65(8):3299-3306.

3. Maynard M, Evans JA, Hosomi T, Hara S, Jewett MA, Ohh M. Human HIF-3alpha4 is a dominant-negative regulator of HIF-1 and is downregulated in renal cell carcinoma. FASEB J. 2005;19(11):1396-1406.

4. Berezikov E, Furyev V, van de Belt J, Wienholds E, Plasterk RH, Cuppen E. Phylogenetic shadowing and computational identification of human microRNA genes. Cell. 2005;120(1):21-24.

5. Sun W, Julie Li YS, Huang HD, Shyy JY, Chien S. microRNA: a master regulator of cellular processes for bioengineering systems. Annu Rev Biomed Eng. 2010;12:1-27.

6. Chan SY, Loscalzo J. MicroRNA-210: a unique and pleiotropic hypoxamir. Cell Cycle. 2010; 9(6):1072-1083

7. Ghosh G, et al. Hypoxia-induced microRNA-424 expression in human endothelial cells regulates HIF- $\alpha$ isoforms and promotes angiogenesis. J Clin Invest. 2010;120(11):4141-4154.

8. Mouillet JF, Chu T, Nelson DM, Mishima T, Sadovsky Y. MiR-205 silences MED1 in hypoxic primary chain trophoblasts. FASEB J. 2010; 24(6):2030-2039.

9. Crosby ME, Devlin CM, Glazer PM, Calin GA, Ivan $\mathrm{M}$. Emerging roles of microRNAs in the molecular responses to hypoxia. Curr Pharm Des. 2009; 15(33):3861-3866.

10. Kulshreshtha R, et al. A microRNA signature of hypoxia. Mol Cell Biol. 2007;27(5):1859-1867.

11. Giannakakis A, et al. miR-210 links hypoxia with cell cycle regulation and is deleted in human epithelial ovarian cancer. Cancer Biol Ther. 2008;7(2):255-264.

12. Fasanaro P, et al. MicroRNA-210 modulates endothelial cell response to hypoxia and inhibits the receptor tyrosine kinase ligand Ephrin-A3. J Biol Chem. 2008;283(23):15878-15883.

13. Crosby ME, Kulshreshtha R, Ivan M, Glazer PM. MicroRNA regulation of DNA repair gene expression in hypoxic stress. Cancer Res. 2009; 69(3):1221-1229.

14. Chan SY, Zhang YY, Hemann C, Mahoney CE,
Zweier JL. MicroRNA-210 controls mitochondrial metabolism during hypoxia by repressing the ironsulfur cluster assembly proteins ISCU $1 / 2$. Cell Metab. 2009;10(4):273-284.

15. Favaro E, et al. MicroRNA-210 regulates mitochondrial free radical response to hypoxia and krebs cycle in cancer cells by targeting iron-sulfur cluster protein ISCU. PLoS One. 2010;5(4):e10345.

16. Semenza GL. Hypoxia-inducible factor 1: Regulator of mitochondrial metabolism and mediator of ischemic preconditioning [published online ahead of print August 21, 2010]. Biochim Biophys Acta. doi: 10.1016/j.bbamcr.2010.08.006.

17. Cascio S, et al. miR20b modulates VEGF expression by targeting HIF-1alpha and STAT3 in MCF-7 breast cancer cells. J Cell Physiol. 2010;224(1):242-249.

18. Rane S, et al. Downregulation of miR-199a derepresses hypoxia-inducible factor-1alpha and Sirtuin 1 and recapitulates hypoxic preconditioning in cardiac myocytes. Circ Res. 2009;104(7):879-886.

19. Yamakuchi M, et al. P-53-induced microRNA-107 inhibits HIF-1 and tumor angiogenesis. Proc Natl Acad Sci U S A. 2010;107(14):6334-6339.

20. TaguchiA, etal. Identification of hypoxia-induciblefactor-1alpha as a novel target for miR-17-92 microRNA cluster. Cancer Res. 2008;68(14):5540-5545.

21. Cha ST, et al. MicroRNA-519c suppresses hypoxiainducible factor-1alpha expression and tumor angiogenesis. Cancer Res. 2010;70(7):2675-2685.

22. Liu CJ, et al. miR31 ablates expression of the HIF regulatory factor FIH to activate the HIF pathway in head and neck carcinoma. Cancer Res. 2010;70(4):1635-1644.

23. Fijalkowska I, et al. Hypoxia-inducible-factor1 alpha regulates the metabolic shift of pulmonary hypertensive endothelial cells. Am J Path. 2010;176(3):1130-1138.

24. Rosa A, et al. The interplay between the master transcription factor PU.1 and miR424 regulates human monocyte/macrophage differentiation. Proc Natl Acad Sci U S A. 2007;104(50):19849-19854.

25 . Forrest $A R$, et al. Induction of microRNAs, miR-155, miR-222, miR-424, and miR-503, promotes monocytic differentiation through combinatorial regulation. Leukemia. 2010;24(2):460-466.

\title{
The magic and mystery of miR-21
}

Edward E. Morrisey

Departments of Medicine and Cell and Developmental Biology, Institute for Regenerative Medicine, Cardiovascular Institute, University of Pennsylvania, Philadelphia, Pennsylvania, USA.

\begin{abstract}
MicroRNAs (miRNAs) are potent regulators of mRNA stability and thereby protein expression. As such, miRNAs have become of interest as possible therapeutics and/or therapeutic targets. In this context, small complementary miRNA sequences known as antagomirs could be used to inhibit miRNA activity, while miRNA mimics could confer gain-of-function activity. However, a note of caution is sounded by Patrick et al. in this issue of the JCI, as they show that although recent reports have suggested that an miR-21 antagomir might be therapeutically useful in preventing heart failure in mice, genetic deletion of miR-21 does not confer a similar phenotype, suggesting possible confounding factors that are only now beginning to be revealed in the techniques used to study miRNA biology.
\end{abstract}

Conflict of interest: The author has declared that no conflict of interest exists.

Citation for this article: $J$ Clin Invest. 2010; 120(11):3817-3819. doi:10.1172/JCI44596.
MicroRNAs (miRNAs) are small, noncoding RNAs transcribed within the introns of other genes or encoded separately as uniquely regulated RNA gene prod- ucts. The approximately 70-bp precursor miRNA product is processed by the enzymes Drosha and Dicer to generate a mature 22-bp product that binds mRNAs in a unique manner using Watson-Crick base pairing through a conserved 6 - to 8-bp "seed" sequence as well as additional contacts (Figure 1). Binding of miRNAs to their target mRNAs inhibits protein translation and reduces mRNA stability.

miRNAs were first identified in the model organism Caenorhabditis elegans but soon after were shown to play potent roles in regulating mRNA stability, and thereby protein expression, in higher eukaryotes. Studies using genetic deletion or inhibi- 